OPEN ACCESS

Edited by:

Mira Katan,

University Hospital Zürich, Switzerland

Reviewed by:

Gian Marco De Marchis, University of Basel, Switzerland

Raffaele Ornello,

University of L'Aquila, Italy

*Correspondence:

Fenghua Jiang

jfhifh2020@126.com

tThese authors have contributed equally to this work

Specialty section:

This article was submitted to

Stroke,

a section of the journa

Frontiers in Neurology

Received: 16 December 2020 Accepted: 23 March 2021

Published: 23 April 2021

Citation:

Wu Q, Cui J, Xie Y, Wang M, Zhang $H$, Hu $X$ and Jiang $F$ (2021) Outcomes of Ischemic Stroke and Associated Factors Among Elderly Patients With Large-Artery Atherosclerosis: A Hospital-Based Follow-Up Study in China. Front. Neurol. 12:642426. doi: 10.3389/fneur.2021.642426

\section{Outcomes of Ischemic Stroke and Associated Factors Among Elderly Patients With Large-Artery Atherosclerosis: A Hospital-Based Follow-Up Study in China}

\author{
Qianqian Wu ${ }^{1+}$, Jingjing Cui ${ }^{1+}$, Yuanli Xie ${ }^{1}$, Min Wang ${ }^{1}$, Huifang Zhang ${ }^{1}$, Xiaofei $\mathrm{Hu}^{1}$ and \\ Fenghua Jiang ${ }^{2 *}$
}

'Department of Neurology, Dongying People's Hospital, Dongying, China, ${ }^{2}$ Department of Rehabilitation Medicine, Dongying People's Hospital, Dongying, China

Large-artery atherosclerotic (LAA) stroke is the most common subtype of ischemic stroke. However, risk factors for long-term outcomes of LAA stroke in the elderly Chinese population have not been well-described. Therefore, we aimed to assess outcomes and risk factors at 3, 12, and 36 months after LAA stroke onset among stroke patients aged 60 years and older. All consecutive LAA patients aged $\geq 60$ years were prospectively recruited from Dongying People's Hospital between January 2016 and December 2018. The clinical features and outcome data at 3, 12, and 36 months after stroke were collected. Differences in outcomes and relationship between outcomes and risk factors were assessed. A total of 1,772 patients were included in our study $(61.7 \%$ male, $38.3 \%$ female). The rates of mortality, recurrence, and dependency were $6.6,12.6$, and $12.6 \%$, respectively, at 3 months after stroke onset. The corresponding rate rose rapidly at 36 months $(23.2,78.7$, and $79.7 \%$, respectively). We found the positive predictors associated outcomes at 3, 12, and 36 months after stroke onset. The relative risk $(\mathrm{RR})$ with $95 \%$ confidential interval $(\mathrm{Cl})$ is $1.06(1.02-1.10$, $P=0.006)$ at 3 months, $1.06(1.02-1.10, P=0.003)$ at12 months, and $1.10(1.05-1.15$, $P<0.001)$ at 36 months after stroke onset for age; $1.09(1.01-1.19, P=0.029)$ at 12 months for fasting plasma glucose (FPG) level; $4.25(2.14-8.43, P<0.001)$ at 3 months, $4.95(2.70-9.10, P<0.001)$ at 12 months, and $4.82(2.25-10.32$, $P<0.001)$ at 36 months for moderate stroke; $7.56(3.42-16.72, P<0.001)$ at 3 months, $11.08(5.26-23.34, P<0.001)$ at 12 months, and 14.30 (4.85-42.11, $P<0.001)$ at 36 months for severe stroke, compared to mild stroke. Hypersensitive C-reactive protein (hs-CRP) level was an independent risk factor for mortality at different follow-up times, with the RR (95\%) of $1.02(1.01-1.02, P<0.001)$ at 3 months, $1.01(1.00-1.02, P=0.002)$ at 12 months. White blood cell count (WBC) level was associated with both stroke recurrence $(\mathrm{RR}=1.09,95 \% \mathrm{Cl}: 1.01-1.18, P=0.023)$ and dependency $(\mathrm{RR}=1.10,95 \% \mathrm{Cl}: 1.02-1.19, P=0.018)$ at 3 months. In contrast, a higher level of low-density lipoprotein cholesterol (LDL-C) within the normal range 
was a protective factor for recurrence and dependency at shorter follow-up times, with the RR (95\%) of $0.67(0.51-0.89, P=0.005)$ and $0.67(0.50-0.88, P=0.005)$, respectively. These findings suggest that it is necessary to control the risk factors of $L A A$ to reduce the burden of LAA stroke. Especially, this study provides a new challenge to explore the possibility of lowering LDL-C level for improved stroke prognosis.

Keywords: outcomes, ischemic stroke, risk factors, elderly, large-artery atherosclerosis

\section{INTRODUCTION}

Stroke affects more than 10 million people worldwide annually and is the second-most common cause of death and the thirdmost common cause of long-term disability (1). Stroke can seriously affect the quality of life of patients and initiates a heavy burden on the families of patients and society (2). Large-artery atherosclerotic (LAA) stroke is the most common subtype of ischemic stroke, especially among Asian population accounting for about 33\%, moreover, LAA increasing fastest among all subtypes reaching $5.7 \%$ annually (3). Compared with other stroke mechanisms, patients with macrovascular atherosclerosis such as internal carotid artery, intracranial vessel, or posterior circulation atherosclerosis had the highest risk of first stroke and early stroke recurrence $(4,5)$.

Underlying mechanisms of stroke caused by large atherosclerosis are diverse, including arterial-to-arterial emboli, in situ thromboembolism, hemodynamic impairment, and branching occlusive disease (BOD), and lesions could be in the lacunae, subcortical, cortical, or these bindings (6). Different studies on the prognosis and risk factors of ischemic stroke subtypes have shown that dyslipidemia, hypertension, diabetes, and obesity affect the prevalence of stroke and stroke-related mortality (7-9). Other traditional cardiovascular risk factors such as sex, race, smoking, alcohol consumption, risk-reducing medication, blood glucose level, and obesity can also increase the progression of atherosclerosis and thus affect the prognosis of this subtype of stroke (10). Furthermore, homocysteine and hs-CRP levels have also predicted stroke-related mortality $(11,12)$.

Although previous studies explored the prognostic outcomes of LAA stroke, few have explored the risk factors that affected the prognosis of this subtype of stroke among elderly patients. Therefore, we aimed to assess the outcomes and their risk factors at 3, 12, and 36 months after LAA stroke onset among stroke patients aged 60 years and older.

\section{MATERIALS AND METHODS}

\section{Participants}

This was a prospective, single-center, hospital-based study that recruited patients from the stroke unit of Dongying People's Hospital, a tertiary general hospital in Shandong province, China, between January 2016 and December 2018, and all information of following-up was collected before January 2020. Consecutive patients who experienced a first LAA ischemic stroke were recruited. Patients who died before the imaging examination, those who experienced transient ischemic attack, and those under 60 years old were excluded.

The study was approved by the ethics committee for medical research at Dongying People's Hospital. Written informed consent was obtained from each participant during recruitment.

\section{LAA Diagnosis Criteria}

We selected LAA stroke [the Trial Org 10172 in Acute Stroke Treatment (TOAST) classification] patients who were diagnosed by clinicians according to the following criteria (13): (a) clinical findings include those of cerebral cortical impairment or brain stem or cerebellar dysfunction; (b) cortical or cerebellar lesions and brainstem or subcortical hemispheric infarcts $>1.5 \mathrm{~cm}$ in diameter on CT or MRI; and (c) supportive evidence by duplex imaging of a stenosis of $>50 \%$ of an appropriate intracranial or extracranial artery.

\section{Definitions of Clinical Features}

Clinical features included stroke severity, stroke risk factors (hypertension, diabetes mellitus, atrial fibrillation, hyperlipidemia, obesity, current smoking, and drinking), neurological function scores [National Institutes of Health Stroke Scale (NIHSS), Barthel Index (BI), and modified Rankin Scale (mRS) scores], and laboratory test values [levels of fasting plasma glucose (FPG), total cholesterol (TC), triglycerides (TG), high-density lipoprotein cholesterol (HDL-C), low-density lipoprotein cholesterol (LDL-C), high-sensitivity hypersensitive C-reactive protein (hs-CRP), white blood cell count (WBC), and blood platelets count (BPC)].

Stroke severity was categorized into three groups according to the National Institutes of Health Stroke Scale (NIHSS) score: mild (NIHSS score: $\leq 7$ ), moderate (NIHSS score: 8-16), and severe (NIHSS score: $\geq 17$ ) (14). Hypertension was defined as systolic blood pressure $\geq 140 \mathrm{mmHg}$, diastolic blood pressure $\geq 90 \mathrm{mmHg}$, or taking medication for hypertension. Diabetes mellitus was defined as a FPG level $\geq 7.0 \mathrm{mmol} / \mathrm{L}$ or taking medication for diabetes. Obesity was defined as a body mass index of $\geq 28.0 \mathrm{~kg} / \mathrm{m}^{2}$. Atrial fibrillation (AF) was defined as defined as a self-reported previous history of AF by patients or the presence of AF detected using 12-lead electrocardiography during hospitalization. Current smoking was defined as smoking $\geq 1$ cigarette per day for more than 1 year and current drinking as drinking alcohol at least once per week for more than 1 year.

\section{Outcome Assessments}

Outcomes included mortality, recurrence, and dependency at 3, 12 , and 36 months after stroke onset. Mortality was defined as allcause cumulative death during the follow-up period. Recurrence 
was defined as new-onset focal or global neurological dysfunction of vascular origin occurring $>30$ days after the initial stroke. Dependency was defined as an mRS score $>2$.

Follow-up evaluations were conducted at 3, 12, and 36 months after stroke onset for all patients by the same trained senior neurologist in a face-to-face interview, except for patients who were re-examined in their local hospitals for whom information was collected by telephone. The investigator contacted the relatives or caregivers of survivors who could not be reached by telephone.

\section{Statistical Analysis}

Continuous variables (age, FPG, TC, TG, hs-CRP, WBC, BPC, HDL-C, and LDL-C) are presented as means and standard deviation (SD). Categorical variables (stroke severity, hypertension, diabetes mellitus, obesity, current smoking, and current drinking) are presented as numbers with frequency. In the univariate analysis, continuous variables were compared with Student's $t$-tests and categorical variables were compared using chi-squared tests. In the multivariate analysis, dependent variables were stroke outcomes at 3,12, and 36 months after stroke onset and the independent variable were variables which $P \leq 0.05$ in the univariate analysis. The results of multivariate analysis were expressed as the adjusted relative risk (RR) and $95 \%$ confidence interval $(\mathrm{CI}) . P$-values $\leq 0.05$ were considered statistically significant. SPSS for Windows (version 22.0; SPSS Inc., Chicago, IL, USA) was used for analyses.

\section{RESULTS}

\section{Baseline Characteristics of Patients With LAA}

In this study, a total of 1,772 patients ( $\geq 60$ years) with LAA ischemic stroke were involved in this follow-up study. The mean age was 70.60 years; $1,094(61.7 \%)$ patients were men, and 678 (38.3\%) were women. According to stroke severity, patients were classified into three grades: $63.2 \%$ mild, $27.1 \%$ moderate, and $9.7 \%$ severe. The prevalence of hypertension, AF, diabetes, and hyperlipidemia at baseline was 75.6, 6.9, 34.0, and 26.2\%, respectively, smoking and drinking rates were 32.8 and $14.7 \%$, respectively. The rate of obesity for these individuals was $13.4 \%$ at baseline. Average laboratory test values were FPG, $6.89 \pm 2.92$ $\mathrm{mmol} / \mathrm{L} ; \mathrm{TC}, 4.92 \pm 1.13 \mathrm{mmol} / \mathrm{L} ; \mathrm{TG}, 1.51 \pm 1.01 \mathrm{mmol} / \mathrm{L}$; HDL-C, $1.09 \pm 0.30 \mathrm{mmol} / \mathrm{L}$; LDL-C, $2.96 \pm 0.88 \mathrm{mmol} / \mathrm{L}$; and $\mathrm{WBC}, 7.78 \pm 2.65 \times 10^{9} / \mathrm{L}$ (Table 1$)$.

Of these patients, there were $93.5 \%(1,657 / 1,772)$, $88.3 \%(1,171 / 1,326)$, and $82.0 \%(341 / 416)$ patients finished the following-up at 3 months, 1 year, and 3 years after stroke, respectively.

\section{Outcomes at 3, 12, and 36 Months After Stroke Onset}

The rates of mortality, recurrence, and dependency rose rapidly after stroke onset. At 3 months after onset, the rates of mortality, recurrence, and dependency were $6.6,12.6$, and $12.6 \%$, respectively, rising to $10.8,34.3$, and $33.0 \%$ at 12 months and 23.2 , 78.7 , and $79.7 \%$ at 36 months (Table 2 ).
TABLE 1 | Baseline characteristics of patients with LAA stroke (age $\geq 60$ years) stratified by sex group.

\begin{tabular}{|c|c|c|c|}
\hline Characteristics & Men & Women & Total \\
\hline Case, $n(\%)$ & $1,094(61.7)$ & 678 (38.3) & 1,772 \\
\hline \multicolumn{4}{|l|}{ Stroke severity, $\boldsymbol{n}(\%)$} \\
\hline Mild & $712(65.1)$ & 407 (60.0) & $1,119(63.2)$ \\
\hline Moderate & $290(26.5)$ & $190(28.0)$ & $480(27.1)$ \\
\hline Severe & $91(8.3)$ & $81(11.9)$ & $172(9.7)$ \\
\hline \multicolumn{4}{|c|}{ Clinical risk factors, $n(\%)$} \\
\hline Hypertension & $774(70.7)$ & 566 (83.5) & $1,340(75.6)$ \\
\hline Diabetes mellitus & $333(30.4)$ & $270(39.8)$ & $603(34.0)$ \\
\hline Atrial fibrillation & $66(6.0)$ & $56(8.3)$ & $122(6.9)$ \\
\hline Hyperlipoidemia & $286(26.1)$ & $178(26.3)$ & $464(26.2)$ \\
\hline Obesity & $100(9.1)$ & $138(20.4)$ & $238(13.4)$ \\
\hline Current smoking & $487(44.5)$ & 95 (14.0) & $582(32.8)$ \\
\hline Current drinking & $250(22.9)$ & $10(1.5)$ & $260(14.7)$ \\
\hline \multicolumn{4}{|c|}{ Laboratory tests, mean (SD) } \\
\hline FPG, mmol/L & $6.71(2.84)$ & $7.16(3.02)$ & $6.89(2.92)$ \\
\hline $\mathrm{TC}, \mathrm{mmol} / \mathrm{L}$ & $4.65(1.01)$ & $5.38(1.18)$ & $4.92(1.13)$ \\
\hline $\mathrm{TG}, \mathrm{mmol} / \mathrm{L}$ & $1.41(0.89)$ & $1.68(1.17)$ & $1.51(1.01)$ \\
\hline HDL-C, mmol/L & $1.05(0.30)$ & $1.16(0.29)$ & $1.09(0.30)$ \\
\hline LDL-C, mmol/L & $2.80(0.80)$ & $3.21(0.95)$ & $2.96(0.88)$ \\
\hline WBC, $10^{9} / \mathrm{L}$ & $7.86(0.73)$ & $7.63(2.51)$ & $7.78(2.65)$ \\
\hline $\mathrm{BPC}, 10^{9} / \mathrm{L}$ & 208.44 (93.32) & 234.73 (71.64) & $218.43(86.65)$ \\
\hline Age, year, mean (SD) & $70.08(7.59)$ & $71.44(7.38)$ & $70.60(7.54)$ \\
\hline \multicolumn{4}{|c|}{ Neurological function, median (range) } \\
\hline NIHSS & $5.00(8)$ & $6.00(8)$ & $6.00(8)$ \\
\hline $\mathrm{Bl}$ & $60.00(50)$ & $55.00(45)$ & $50.00(50)$ \\
\hline $\mathrm{mRs}$ & $3.00(2)$ & $3.00(2)$ & $3.00(2)$ \\
\hline
\end{tabular}

\section{Univariate Factors Associated With Stroke-Related Outcomes}

Table 2 shows that stroke severity, FPG level, hs-CRP level, WBC, and age were significantly correlated with 3-, 12-, and 36-month mortality (all $P<0.001$ at 3 - and 12-month; $P<0.001, P=0.005$, $P<0.001, P<0.001$, and $P<0.001$ at 36 -month). Smoking, drinking, and AF were associated with 3 - and 12-month mortality ( $P=0.025, P=0.024$, and $P=0.009$ at 3 -month; $P=0.007$, $P=0.010$, and $P=0.001$ at 12 -month). Moreover, TC, HDL$C$, and LDL-C levels were correlated with 36 -month mortality ( $P=0.028, P=0.004$, and $P=0.050$, respectively).

Stroke severity, hs-CRP level, WBC, and age were correlated with 3 - and 12-month recurrence $(P<0.001, P=0.005$, $P=0.004$, and $P=0.010$ at 3 -month; $P<0.001, P=0.001$, $P=0.002$, and $P<0.001$ at 12 -month). FPG and HDL-C, and LDL-C were correlated with 3-month recurrence. Moreover, AF was associated with 12 -month recurrence $(P=0.043)$. However, no risk factors were found associated with recurrence at 36 months after stroke onset.

Stroke severity was correlated with 3-, 12-, and 36-month dependence (all $P<0.001$ ). Hypersensitive CRP level, WBC, and age were associated with 3 - and 12-month dependence ( $P=0.005, P=0.003$, and $P=0.007$ at 3 -month; $P=0.002$, 
TABLE 2 | Factors associated with outcomes at 3-, 12-, and 36-month after stroke among patients with LAA by univariate analysis.

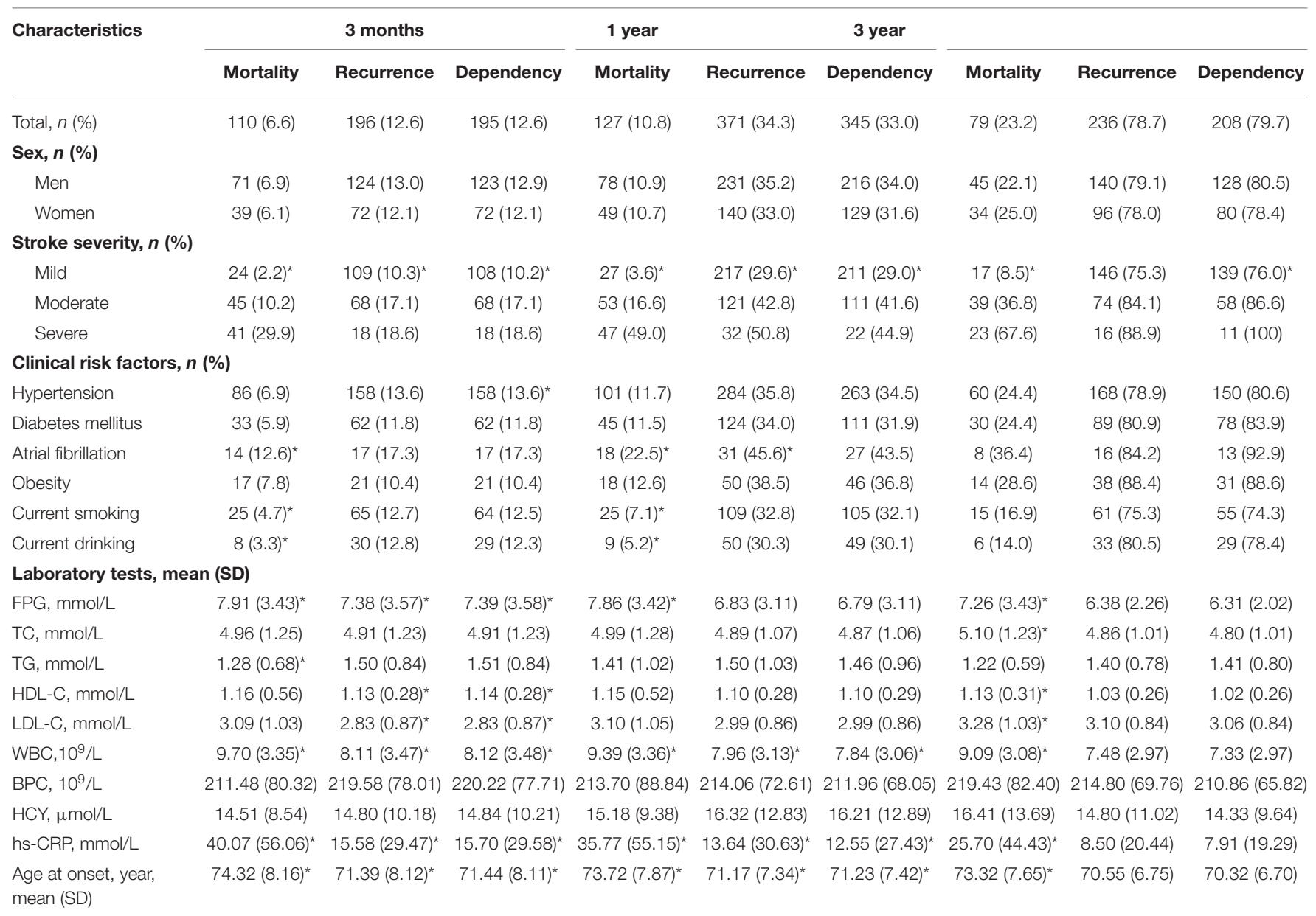

${ }^{*} P<0.05$.

$P=0.018$, and $P<0.001$ at 12 -month). Hypertension and FPG, HDL-C, and LDL-C levels were associated with 3month dependence $(P=0.039, P=0.004, P=0.013$, and $P=0.044$, respectively).

\section{Predictors of Outcomes in the Multivariate Analysis Mortality}

In the multivariate analysis, both stroke severity and older age were associated with higher risk of mortality at 3, 12, and 36 months after stroke onset. Compared with mildly affected stroke patients, severely affected stroke increased mortality risk 6.56fold (95\% CI: 3.42-16.72, $P<0.001$ ) at 3 months and 13.3fold (95\% CI: 4.85-42.11, $P<0.001$ ) at 36 months after stroke onset. For each 1-year increased in age, mortality risk increased $6 \%$ at 3 months (95\% CI: $1.02-1.10, P=0.006)$ and $10 \%$ at 36 months after stroke onset. Moreover, AF increased 1.77-fold mortality risk (95\% CI: $1.29-5.94, P=0.009)$ only at 12 months. In addition, higher FPG (95\% CI: $1.01-1.19, P=0.029)$ and hsCRP (95\% CI: $1.00-1.02, P=0.002)$ levels were also risk factors of mortality at 12 months after stroke onset (Figure 1).

\section{Recurrence}

The hazard of mortality 3 months after stroke was associated with a higher FPG level (RR $=1.11,95 \% \mathrm{CI}: 1.04-1.18, P=0.001)$, LDL-C level ( $\mathrm{RR}=0.67,95 \% \mathrm{CI}: 0.51-0.89, P=0.005)$, WBC $(\mathrm{RR}=1.09,95 \% \mathrm{CI}: 1.01-1.18, P=0.023)$, and older age $(\mathrm{RR}=1.06,95 \% \mathrm{CI}: 1.02-1.09, P=0.001)$. The risk of 12 -month recurrence in moderately affected and severely affected patients were 1.59-fold (95\% CI: 1.12-2.25, $P=0.009$ ) and 2-fold higher (95\% CI: $1.07-3.74, P=0.030$ ) than in mildly affected patients. For each $1 \times 10^{9} / \mathrm{L}$ increase in $\mathrm{WBC}$, the risk of recurrence increased 6\% (95\% CI: 1.00-1.13, P = 0.050); for each 1-year increase in age, the risk of recurrence increased 3\% (95\% CI: 1.01-1.05, $P=0.005$ ) (Figure 2).

\section{Dependence}

For each unit increase in FPG and LDL-C levels and WBC, the risk of 3-month dependency increased 11\% (95\% CI: 1.04-1.18, $P=0.001)$, decreased 33\% (95\% CI: 0.50-0.88, $P=0.005)$, and increased $10 \%$ (95\% CI: $1.02-1.19, P=0.018)$, respectively. The risk of 12-month dependency in moderately affected patients was $54 \%$ higher than in mildly affected patients (95\% CI: 1.08-2.20, $P=0.018$ ). Moreover, older age increased the risk of dependency 


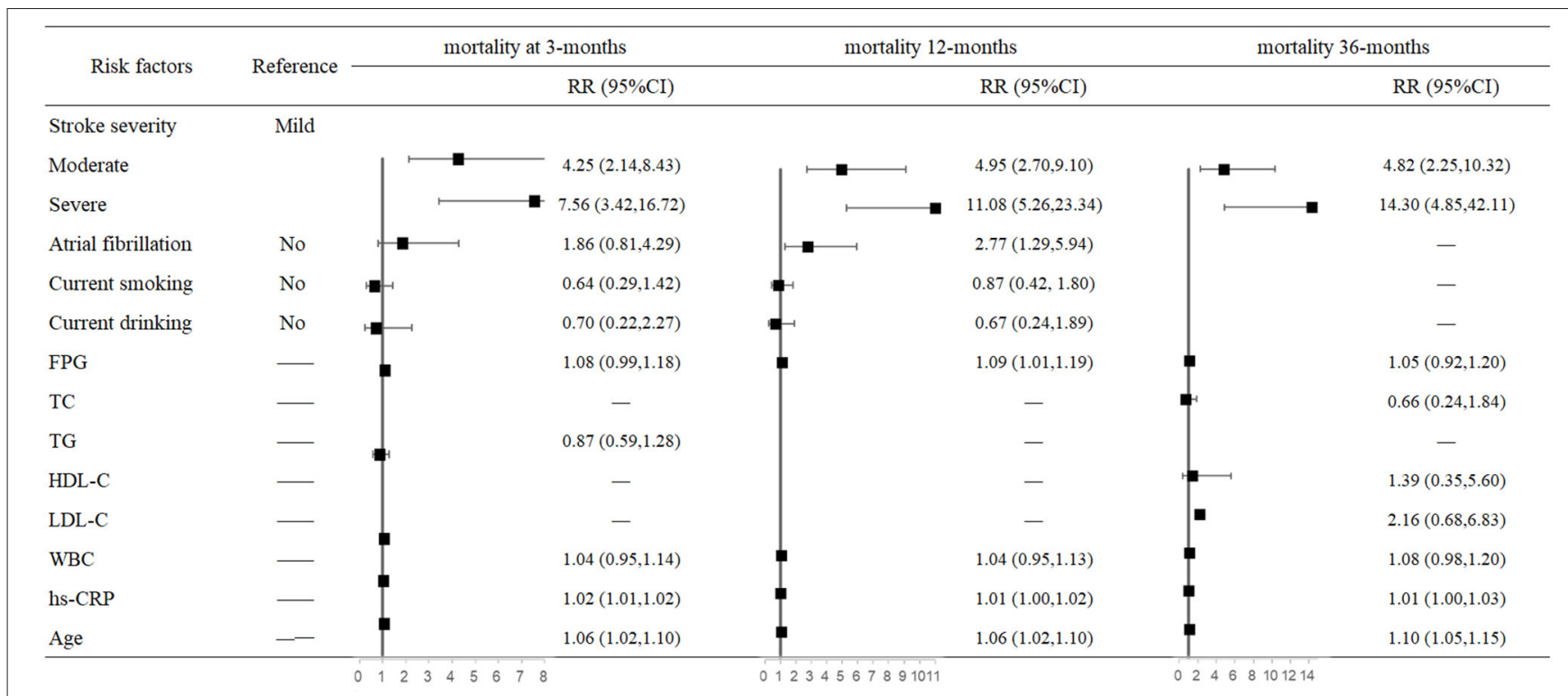

FIGURE 1 | Predictors of mortality at 3, 12, and 36 months after stroke in the multivariate analysis.

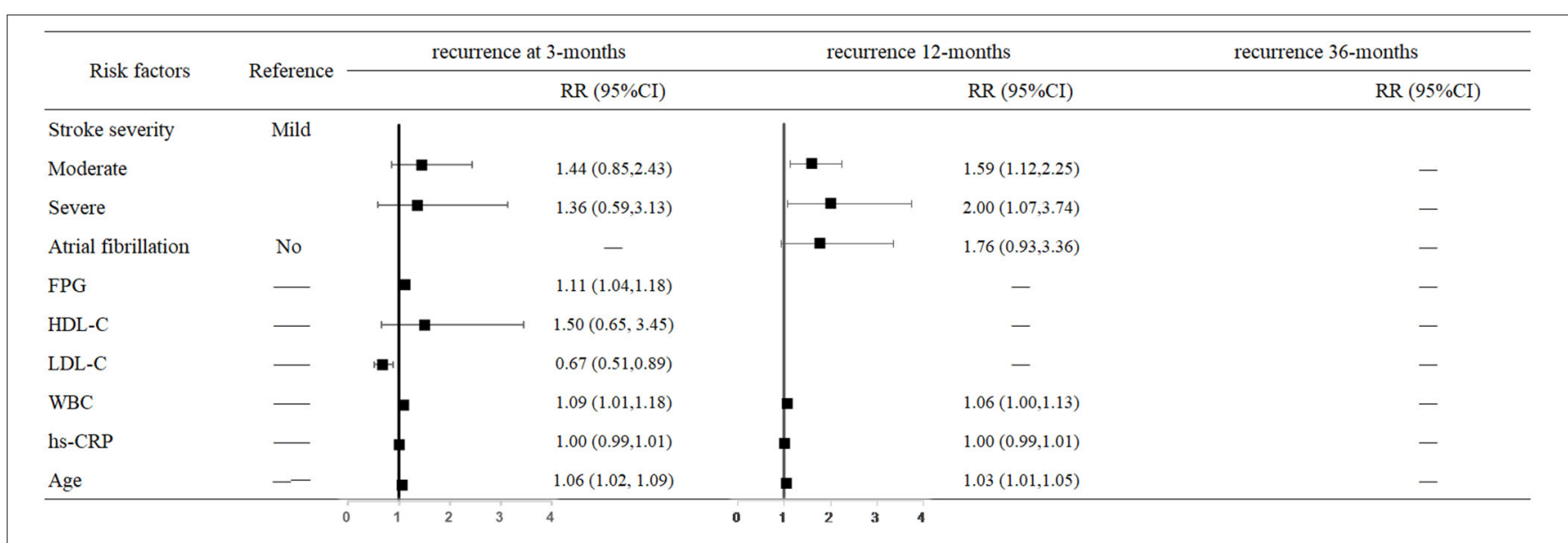

FIGURE 2 | Predictors of recurrence at 3, 12, and 36 months after stroke in the multivariate analysis.

at both 3 months $(\mathrm{RR}=1.06,95 \% \mathrm{CI}: 1.03-1.10, P<0.001)$ and 12 months $(\mathrm{RR}=1.04,95 \% \mathrm{CI}: 1.01-1.06, P=0.002)$ after stroke onset (Figure 3).

\section{DISCUSSION}

This was a hospital-based follow-up study that explored outcomes and associated risk factors of LAA stroke in China in patients aged 60 years and older. The rates of mortality, recurrence, and dependency all dramatically increased between 3 and 36 months after stroke onset. Factors associated with this increase included older age, higher FPG levels, and stroke severity. Moreover, hs-CRP was an independent risk factor for mortality at 12 months after stroke onset. Furthermore, WBC level was associated with both stroke recurrence and dependency. In contrast, a higher LDL-C level was a protective factor for recurrence and dependency in short times.

Different cohort studies and population studies in Africa, such as TSIP, INTERSTROKE, and SIREN, have shown that older age was the strongest, immutable risk factor for stroke, and similar results have been reported for developed countries (15). Stroke involves cerebral vessel arteriosclerosis, which is a silent progressive process that can begin early in life and usually develops with age (16). Two hospital-based studies have shown that age was an important inverse predictor of functional recovery after ischemic stroke for 3 months, independent of stroke severity, characteristics, and complications, while the association of age with prognostic outcome is non-linear and extends to young stroke patients $(17,18)$. Other studies that 


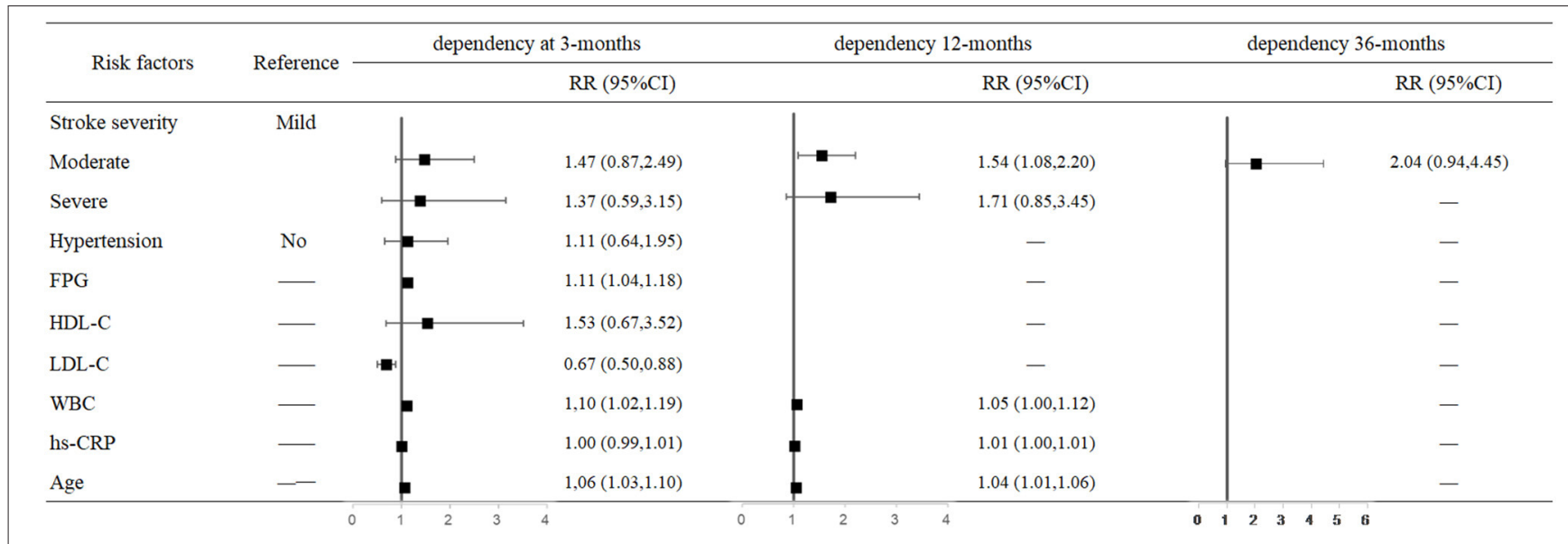

FIGURE 3 | Predictors of dependency at 3, 12, and 36 months after stroke in the multivariate analysis.

examined risk factors for stroke subtypes based on the TOAST classification revealed that age was independently associated with short term (90-day) mortality in patients with LAA stroke $(2,19)$. The Atherosclerosis Risk in Communities (ARIC) study also reported that age was an independent risk factor for nonlacunar ischemic stroke subtypes after long term follow-up (20). Consistent with previous results, our study also clearly showed that older age increased the risk of outcomes at short and long term after the onset of LAA stroke, further validating the effect of age on the prognosis of this subtype of stroke.

Stroke severity was another important risk factor for stroke outcomes. One clinical trial included 1,281 stroke patients and used the TOAST classification to classify stroke and NIHSS to quantify neurological impairment at baseline. The results showed that NIHSS scores strongly predicted the likelihood of recovery in patients after stroke. Scores $\geq 16$ indicated higher likelihood of death or severe disability, while score $\leq 6$ indicated good recovery (21). A study in Italy also showed that NIHSS score was an important predictor of functional decline at 3 months (17). A study of gender differences in China also found that stroke severity was an independent risk factor for mortality, dependence, and recurrence in men and women at 3 and 12 months after stroke (22). In our study, the results showed an increased risk of prognosis (mortality, recurrence, and dependence) in moderate and severe relative to mild stroke over three periods. These results all confirmed that baseline NIHSS scores could be used as predictors of outcome after stroke.

Previous studies reported that high FPG level was correlated with poor outcomes of ischemic stroke (23-25). A study reported that acute ischemic stroke patients with a favorable neurologic outcome (90-day mRS scores $\leq 2$ ) had a significantly lower baseline FPG level than those with an unfavorable neurologic outcome ( $6.6 \pm 1.96$ vs. $8.12 \pm 4.02 ; P=0.002)$ (24). The 6.5year prospective Northern Manhattan Study (NOMAS) reported that diabetic subjects with elevated FPG level [HR 2.7 (95\% CI 2.0-3.8)] were at increased risk of stroke, but those with a target FPG level $(<7.0 \mathrm{mmol} / \mathrm{L})$ were not, even after adjustment (26).
Patients with type 2 diabetes were more likely to suffer from obesity, transient ischemic attack, and stroke caused by LAA and had a worse prognosis than in patients without diabetes (27). Moreover, increased FPG level on admission has been associated with poorer functional outcomes, irrespective of diabetes status after an acute ischemic stroke (28-30). Another study showed that the risk of ischemic stroke increased by $8.5 \%$ for each 1 $\mathrm{mmol} / \mathrm{L}$ increase in FPG level, even in non-diabetic patients (23). The results of the present study showed that elevated FPG level was an independent risk factor for 3- and 12-month outcomes, further illustrating that high FPG level is a strong prediction factor for poor outcomes of LAA stroke; accordingly, early intervention and management should be considered to improve LAA stroke prognosis.

Increasing evidence suggests that inflammation is a key process in the pathogenesis of atherosclerosis. After acute stroke, CRP level is elevated due to tissue injury and infection, proportional to the severity of acute events. In addition, CRP level had the greatest increase in patients with LAA stroke, and baseline CRP level can independently predict the risk of stroke recurrence (31). Furthermore, serum hs-CRP level was associated with morphological characteristics of rapidly progressing carotid atherosclerosis, suggesting that hs-CRP level was a sensitive marker of the presence of active atherosclerotic disease (32). Furthermore, in a case-control study, serum CRP level in patients with all ischemic stroke subtypes were significantly higher in both the acute phase and at 3-month follow-up (33). Rost et al. found that high serum CRP level can predict the future risk of ischemic stroke in elderly patients (34). Another marker of inflammatory response is the leukocyte level. Compared with the quartile with lowest leukocyte counts at baseline, patients in the top quartile had higher risks for ischemic stroke (35). Evaluating WBC level was also related with both poor shortand long-term outcomes $(36,37)$. The association was strongest for those with LAA stroke compared with other subtypes (adjusted hazard ratio 1.48, 0.98-2.24) (38). The present results further suggest that CRP is an independent risk factors for 
mortality (both short- and long-term) and that WBC level is associated with both stroke recurrence and dependency, adding new evidence for the role of CRP and WBC level in stroke prognosis.

The relationships between LDL-C levels and stroke outcomes remain controversial. A study explored a population with higher LDL cholesterol that was $>15$ times more likely to have ischemic stroke than in a control group (39). Similarly, another showed that a LDL-C level of $\geq 2.6 \mathrm{mmol} / \mathrm{L}$ at admission was an independent risk factor for intracranial atherosclerotic stroke (9). In contrast, other studies reported no clear relationship between LDL-C and ischemic stroke $(40,41)$. Moreover, in contrast to the present results, a multi-center retrospective study revealed that compared with lower LDL-C levels, levels in the third quartile were less likely to exhibit unfavorable outcomes after ischemic stroke (42). Another national cohort study also supports this point, reporting that among the middle-aged and elderly Chinese population, a lower LDL-C level was associated with increased all-cause mortality risk (43). The current results suggest that LDL-C is a protective factor for short-term recurrence and dependence after stroke onset. Regardless, the average LDL$\mathrm{C}$ level at baseline was classified as normal $(<3.36 \mathrm{mmol} / \mathrm{L})$ according to NCEP ATP III criteria (44). It may be that among patients with normal LDL-C levels, a higher level may be negatively associated with stroke outcomes: this hypothesis requires further prospective and well-designed studies for validation.

There are several limitations in the current study. First, the data in our study were collected from a single center in northern China, which may limit the generalization of our results. Moreover, patients recruited in our study were limited 60 years and older, comparisons between the present study and previous ones are limited by the age. Third, all the influencing factors were collected and measured at admission, and no follow-up information was available. Third, we were unable to analyze the impact of other risk factors such as medication adherence after discharge for prognosis. However, these factors have little impact on our current findings. Future studies need to refine information concerning changes in risk factors and drug administration during follow-up. Finally, we did not collect

\section{REFERENCES}

1. Wafa HA, Wolfe C, Bhalla A, Wang Y. Long-term trends in death and dependence after ischaemic strokes: a retrospective cohort study using the South London Stroke Register (SLSR). PLoS Med. (2020) 17:e1003048. doi: 10.1371/journal.pmed.1003048

2. Wei W, Li S, San F, Zhang S, Shen Q, Guo J, et al. Retrospective analysis of prognosis and risk factors of patients with stroke by TOAST. Medicine (Baltimore). (2018) 97:e0412. doi: 10.1097/MD.0000000000010412

3. Ornello R, Degan D, Tiseo C, Di Carmine C, Perciballi L, Pistoia F, et al. Distribution and temporal trends from 1993 to 2015 of ischemic stroke subtypes: a systematic review and meta-analysis. Stroke. (2018) 49:814-19. doi: 10.1161/STROKEAHA.117.020031

4. Starostka-Tatar A, Łabuz-Roszak B, Skrzypek M, Lasek-Bal A, Gasior M, Gierlotka M. Characteristics of hospitalizations due to acute stroke in the information about lipid lowering drugs, which may affect our evaluation on the association between LDL-C and outcomes.

\section{CONCLUSION}

In conclusion, this was a hospital-based study that explored outcomes and associated risk factors among old Chinese LAA stroke patients. Older age, higher FPG level, and stroke severity were independent risk factors for mortality, recurrence, and dependence in LAA stroke. In addition, WBC was a risk factor for recurrence and dependency. Moreover, higher LDL-C level may reduce the risk of short-term recurrence and dependence following stroke in China. Therefore, it is necessary to control risk factors for LAA to reduce the burden of LAA stroke. In particular, this study provides a new challenge to determine whether lowering LDL-C level may improve stroke prognosis.

\section{DATA AVAILABILITY STATEMENT}

The raw data supporting the conclusions of this article will be made available by the authors, without undue reservation.

\section{ETHICS STATEMENT}

The studies involving human participants were reviewed and approved by The Ethics Committee for medical research at Dongying People's Hospital. The patients/participants provided their written informed consent to participate in this study.

\section{AUTHOR CONTRIBUTIONS}

FJ contributed to the study design, performed data collection, data interpretation, critical review, and performed data analysis. QW and JC contributed to drafting of the article. QW, JC, YX, $\mathrm{MW}, \mathrm{HZ}$, and XH performed data collection, case diagnoses, and confirmation of case diagnoses. All authors read, revised, and approved the final version of the paper.

\section{ACKNOWLEDGMENTS}

We thank all participants for their efforts in this study.
Silesian Province, Poland, between 2009 and 2015. Neurol Neurochir Pol. (2018) 52:252-62. doi: 10.1016/j.pjnns.2017.11.010

5. Lange MC, Ribas G, Scavasine V, Ducci RS-P, Mendes DC, Zétola VHF, et al. Stroke recurrence in the different subtypes of ischemic stroke. The importance of the intracranial disease. Arq Neuropsiquiatr. (2018) 76:649-53. doi: 10.1590/0004-282x20180095

6. Marulanda-Londoño E, Chaturvedi S. Stroke due to large vessel atherosclerosis: five new things. Neurol Clin Pract. (2016) 6:252-8. doi: 10.1212/CPJ.0000000000000247

7. Cantú-Brito C, Ruiz-Sandoval JL, Murillo-Bonilla LM, Chiquete E, LeónJiménez C, Arauz A, et al. Acute care and one-year outcome of Mexican patients with first-ever acute ischemic stroke: the PREMIER study. Rev Neurol. (2010) 51:641-9.

8. Deleu D, Inshasi J, Akhtar N, Ali J, Vurgese T, Ali S, et al. Risk factors, management and outcome of subtypes of ischemic stroke: a 
stroke registry from the Arabian Gulf. J Neurol Sci. (2011) 300:142-7. doi: 10.1016/j.jns.2010.08.023

9. Lei C, Wu B, Liu M, Chen Y. Risk factors and clinical outcomes associated with intracranial and extracranial atherosclerotic stenosis acute ischemic stroke. J Stroke Cerebrovasc Dis. (2014) 23:1112-7. doi: 10.1016/j.jstrokecerebrovasdis.2013.09.024

10. Qu B, Qu T. Causes of changes in carotid intima-media thickness: a literature review. Cardiovasc Ultrasound. (2015) 13:46. doi: 10.1186/s12947-01 $5-0041-4$

11. Shi Z, Guan Y, Huo YR, Liu S, Zhang M, Lu H, et al. Elevated total homocysteine levels in acute ischemic stroke are associated with longterm mortality. Stroke. (2015) 46:2419-25. doi: 10.1161/STROKEAHA.115. 009136

12. Xie D, Hu D, Zhang Q, Sun Y, Li J, Zhang Y. Increased high-sensitivity C-reactive protein, erythrocyte sedimentation rate and lactic acid in stroke patients with internal carotid artery occlusion. Arch Med Sci. (2016) 12:54651. doi: 10.5114/aoms.2014.47879

13. Adams HP, Jr., Bendixen BH, Kappelle LJ, Biller J, Love BB, Gordon DL, et al. Classification of subtype of acute ischemic stroke. Definitions for use in a multicenter clinical trial. TOAST. Trial of Org 10172 in Acute Stroke Treatment. Stroke. (1993) 24:35-41. doi: 10.1161/01.STR. 24.1.35

14. Kim JS, Lee KB, Roh H, Ahn MY, Hwang HW. Gender differences in the functional recovery after acute stroke. J Clin Neurol. (2010) 6:183-8. doi: $10.3988 / j \mathrm{jcn} .2010 .6 .4 .183$

15. Akinyemi RO, Owolabi MO, Ihara M, Damasceno A, Ogunniyi A, Dotchin C, et al. Stroke, cerebrovascular diseases and vascular cognitive impairment in Africa. Brain Res Bull. (2019) 145:97-108. doi: 10.1016/j.brainresbull.2018.05.018

16. Scheen AJ. From atherosclerosis to atherothrombosis: from a silent chronic pathology to an acute critical event. Rev Med Liege. (2018) 73:224-8.

17. Turcato G, Cervellin G, Cappellari M, Bonora A, Zannoni M, Bovi P, et al. Early function decline after ischemic stroke can be predicted by a nomogram based on age, use of thrombolysis, RDW and NIHSS score at admission. J Thromb Thrombolysis. (2017) 43:394-400. doi: 10.1007/s11239-01 6-1456-y

18. Knoflach M, Matosevic B, Rücker M, Furtner M, Mair A, Wille G, et al. Functional recovery after ischemic stroke-a matter of age: data from the Austrian Stroke Unit Registry. Neurology. (2012) 78:279-85. doi: 10.1212/WNL.0b013e3182 4367ab

19. Soriano-Tárraga C, Giralt-Steinhauer E, Mola-Caminal M, Ois A, RodríguezCampello A, Cuadrado-Godia E, et al. Biological age is a predictor of mortality in ischemic stroke. Sci Rep. (2018) 8:4148. doi: 10.1038/s41598-018-2 2579-0

20. Ohira T, Shahar E, Chambless LE, Rosamond WD, Mosley TH, Jr., Folsom AR. Risk factors for ischemic stroke subtypes: the atherosclerosis risk in communities study. Stroke. (2006) 37:2493-8. doi: 10.1161/01.STR.0000239694.1 9359.88

21. Adams HP, Jr., Davis PH, Leira EC, Chang KC, Bendixen BH, Clarke WR, et al. Baseline NIH stroke scale score strongly predicts outcome after stroke: a report of the trial of org 10172 in acute stroke treatment (TOAST). Neurology. (1999) 53:126-31. doi: 10.1212/WNL. 53.1 .126

22. Zou C, Wei C, Wang Z, Jin Y. Sex differences in outcomes and risk factors among elderly patients with ischemic stroke. Oncotarget. (2017) 8:104582-93. doi: 10.18632/oncotarget.21967

23. Xing L, Liu S, Tian Y, Yan H, Jing L, Chen K, et al. C-R relationship between fasting plasma glucose and unfavorable outcomes in patients of ischemic stroke without diabetes. I Stroke Cerebrovasc Dis. (2019) 28:1400-8. doi: 10.1016/j.jstrokecerebrovasdis.2019. 02.009

24. Wang F, Jiang B, Kanesan L, Zhao Y, Yan B. Higher admission fasting plasma glucose levels are associated with a poorer short-term neurologic outcome in acute ischemic stroke patients with good collateral circulation. Acta Diabetol. (2018) 55:703-14. doi: 10.1007/s00592-01 8-1139-6

25. Yao T, Zhan Y, Shen J, Xu L, Peng B, Cui Q, et al. Association between fasting blood glucose and outcomes and mortality in acute ischaemic stroke patients with diabetes mellitus: a retrospective observational study in Wuhan, China. BMJ Open. (2020) 10:e037291. doi: 10.1136/bmjopen-2020037291

26. Boden-Albala B, Cammack S, Chong J, Wang C, Wright C, Rundek $\mathrm{T}$, et al. Diabetes, fasting glucose levels, and risk of ischemic stroke and vascular events: findings from the Northern Manhattan study (NOMAS). Diabetes Care. (2008) 31:1132-7. doi: 10.2337/dc 07-0797

27. Putaala J, Liebkind R, Gordin D, Thorn LM, Haapaniemi E, Forsblom C, et al. Diabetes mellitus and ischemic stroke in the young: clinical features and long-term prognosis. Neurology. (2011) 76:1831-7. doi: 10.1212/WNL.0b013e31821cccc2

28. Mandava P, Martini SR, Munoz M, Dalmeida W, Sarma AK, Anderson JA, et al. Hyperglycemia worsens outcome after rt-PA primarily in the large-vessel occlusive stroke subtype. Transl Stroke Res. (2014) 5:519-25. doi: 10.1007/s12975-014-0338-x

29. Masrur S, Cox M, Bhatt DL, Smith EE, Ellrodt G, Fonarow GC, et al. Association of acute and chronic hyperglycemia with acute ischemic stroke outcomes post-thrombolysis: findings from get with the guidelinesstroke. J Am Heart Assoc. (2015) 4:e002193. doi: 10.1161/JAHA.115. 002193

30. Gofir A, Mulyono B, Sutarni S. Hyperglycemia as a prognosis predictor of length of stay and functional outcomes in patients with acute ischemic stroke. Int J Neurosci. (2017) 127:923-9. doi: 10.1080/00207454.2017.12 80793

31. Kelly PJ, Murphy S, Coveney S, Purroy F, Lemmens R, Tsivgoulis G, et al. Antiinflammatory approaches to ischaemic stroke prevention. J Neurol Neurosurg Psychiatry. (2018) 89:211-8. doi: 10.1136/jnnp-2016-314817

32. Schillinger M, Exner M, Mlekusch W, Sabeti S, Amighi J, Nikowitsch R, et al. Inflammation and carotid artery-risk for atherosclerosis study (ICARAS). Circulation. (2005) 111:2203-9. doi: 10.1161/01.CIR.0000163569.97918.C0

33. Ladenvall C, Jood K, Blomstrand C, Nilsson S, Jern C, Ladenvall P. Serum C-reactive protein concentration and genotype in relation to ischemic stroke subtype. Stroke. (2006) 37:2018-23. doi: 10.1161/01.STR.0000231872.86071.68

34. Rost NS, Wolf PA, Kase CS, Kelly-Hayes M, Silbershatz H, Massaro JM, et al. Plasma concentration of C-reactive protein and risk of ischemic stroke and transient ischemic attack: the Framingham study. Stroke. (2001) 32:2575-9. doi: 10.1161/hs1101.098151

35. Grau AJ, Boddy AW, Dukovic DA, Buggle F, Lichy C, Brandt T, et al. Leukocyte count as an independent predictor of recurrent ischemic events. Stroke. (2004) 35:1147-52. doi: 10.1161/01.STR.0000124122.71702.64

36. Quan K, Wang A, Zhang X, Wang Y. Leukocyte count and adverse clinical outcomes in acute ischemic stroke patients. Front Neurol. (2019) 10:1240. doi: 10.3389/fneur.2019.01240

37. Huang WY, Peng TI, Weng WC, Chien YY, Wu CL, Lee M, et al. Higher leukocyte count is associated with higher risk of 3-year mortality in nondiabetic patients with first-ever ischemic stroke. J Neurol Sci. (2012) 316:93-8. doi: 10.1016/j.jns.2012.01.018

38. Elkind MS, Sciacca RR, Boden-Albala B, Rundek T, Paik MC, Sacco RL Relative elevation in baseline leukocyte count predicts first cerebral infarction. Neurology. (2005) 64:2121-5. doi: 10.1212/01.WNL.0000165989.12122.49

39. Biswas N, Sangma MA. Serum LDL (low density lipoprotein) as a risk factor for ischemic stroke. Mymensingh Med J. (2016) 25:425-32.

40. Shahar E, Chambless LE, Rosamond WD, Boland LL, Ballantyne CM, McGovern PG, et al. Plasma lipid profile and incident ischemic stroke: the atherosclerosis risk in communities (ARIC) study. Stroke. (2003) 34:623-31. doi: 10.1161/01.STR.0000057812.51734.FF

41. Li W, Liu M, Wu B, Liu H, Wang LC, Tan S. Serum lipid levels and 3-month prognosis in Chinese patients with acute stroke. Adv Ther. (2008) 25:329-41. doi: 10.1007/s12325-008-0045-7

42. Zhao S, Tian Y, Yan H, Zhang B, Li J, Xing L, et al. Association between lowdensity lipoprotein cholesterol (LDL-C) level and unfavorable outcomes in 
participants of ischemic stroke without diabetes: a multi-center retrospective study. Med Sci Monit. (2019) 25:5934-41. doi: 10.12659/MSM.917798

43. Zhou L, Wu Y, Yu S, Shen Y, Ke C. Low-density lipoprotein cholesterol and allcause mortality: findings from the China health and retirement longitudinal study. BMJ Open. (2020) 10:e036976. doi: 10.1136/bmjopen-2020-036976

44. Expert Panel on Detection, Evaluation, and Treatment of High Blood Cholesterol in Adults. Executive summary of the third report of the national cholesterol education program (NCEP) expert panel on detection, evaluation, and treatment of high blood cholesterol in adults (Adult Treatment Panel III). JAMA. (2001) 285:2486-97. doi: 10.1001/jama.285. 19.2486
Conflict of Interest: The authors declare that the research was conducted in the absence of any commercial or financial relationships that could be construed as a potential conflict of interest.

Copyright $\odot 2021 \mathrm{Wu}, \mathrm{Cui}$, Xie, Wang, Zhang, Hu and Jiang. This is an open-access article distributed under the terms of the Creative Commons Attribution License (CC $B Y)$. The use, distribution or reproduction in other forums is permitted, provided the original author(s) and the copyright owner(s) are credited and that the original publication in this journal is cited, in accordance with accepted academic practice. No use, distribution or reproduction is permitted which does not comply with these terms. 